\section{Intervenções não farmacológicas para manejo do ganho de peso em pacientes com esquizofrenia em uso de antipsicóticos}

\author{
Non-pharmacological interventions for weight gain in \\ patients with schizophrenia taking antipsychotics
}

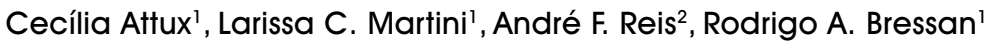

\begin{abstract}
RESUMO
Introdução: Pacientes com esquizofrenia têm maior prevalência de obesidade em comparação à população geral. Esse fato está relacionado a uma alimentação inadequada, ao sedentarismo e ao uso de antipsicóticos. O aumento da obesidade associa-se a diversos distúrbios metabólicos, como o diabetes melito. As intervenções para prevenção e controle do ganho de peso são necessárias nessa população, em especial as intervenções não farmacológicas. Objetivo: Revisar os estudos sobre intervenções não farmacológicas para prevenção e controle do ganho de peso em pacientes com esquizofrenia. Métodos: Foram encontrados oito estudos controlados e quatro não controlados sobre intervenções não farmacológicas. Foi feita uma revisão sobre a metodologia e os fatores positivos e limitações dos estudos. Conclusões: As intervenções não farmacológicas parecem ter um efeito importante em termos de prevenção e controle do ganho de peso e, portanto, devem ser incentivadas e adaptadas à realidade dos pacientes e serviços de saúde. Arq Bras Endocrinol Metab. 2009;53(4):391-8.
\end{abstract}

Descritores

Ganho de peso; esquizofrenia; antipsicóticos; atividade física; nutrição

\begin{abstract}
Introduction: Schizophrenic patients have a higher prevalence of obesity than the general population. There are several factors implicated in weight gain, including poor dietary conditions, sedentary lifestyle and antipsychotic drugs use. Obesity is also associated with metabolic disturbances such as diabetes mellitus. Weight gain interventions are necessary in this population, especially non-pharmacological interventions. Objective: To review the non-pharmacological interventions for weight gain management in patients with schizophrenia. Methods: Eight clinical trials and four open-label studies using these interventions were found. The methodology, strength and limitations of the studies were reviewed. Conclusions: Non-pharmacological interventions seem to have an important effect on weight gain prevention and control, and should be encouraged and adapted to patients and in mental health institution's reality. Arq Bras Endocrinol Metab. 2009;53(4):391-8.
\end{abstract}

Keywords

Weight gain; schizophrenia; antipsychotics; physical activity; nutrition
1 Programa de Esquizofrenia (PROESQ), Departamento de Psiquiatria, Universidade Federal de São Paulo (Unifesp), São Paulo, SP, Brasil

${ }^{2}$ Departamento de Medicina, Disciplina de Endocrinologia, Unifesp, São Paulo, SP, Brasil
Correspondência para: Cecília Attux

Rua Borges Lagoa, 564, cj. 23, Vila Clementino 04038-000 - São Paulo, SP, Brasil cattux@gmail.com

Recebido em 24/Mar/2009 Aceito em 13/Mai/2009

\section{INTRODUÇÃO}

$\mathrm{P}$ acientes com transtorno psicótico, em especial esquizofrenia, têm probabilidade maior de apresentar obesidade ou sobrepeso quando comparados à população geral (1-3). A obesidade nos pacientes com esquizofrenia pode levar à perda da autoestima, ao isolamento social $\mathrm{e}$ 
à estigmatização $(4,5)$. Além disso, a obesidade está fortemente associada a diabetes melito, hipertensão arterial, dislipidemia e consequente doença cardiovascular (6). $\mathrm{O}$ impacto econômico da obesidade também é considerável: estimativas de países desenvolvidos apontam os custos com obesidade como um dos mais altos, em torno de $6 \%$ ou mais das despesas totais com saúde (7).

Em relação à fisiopatologia da obesidade, alguns aspectos devem ser considerados. O ganho de peso está ligado à diminuição da taxa metabólica, ao aumento do influxo calórico e à diminuição da atividade física (8). Sedentarismo, dieta não balanceada e uso de certos antipsicóticos podem contribuir para o ganho de peso e são condições comuns em pacientes com esquizofrenia.

Os antipsicóticos foram um dos principais determinantes para a evolução do tratamento da esquizofrenia, prevenindo e tratando os sintomas e favorecendo a reinserção dos pacientes na comunidade (9). Com o advento da segunda geração de antipsicóticos (atípi$\cos$ ), seu uso tornou-se mais efetivo, com ocorrência menor de sintomas extrapiramidais, além de menor interferência nos níveis circulantes de hormônios como os da prolactina. Entretanto, alguns desses antipsicóticos de segunda geração, tais como olanzapina e clozapina, estão associados ao ganho de peso, à dislipidemia e à hipertensão arterial, com elevado risco para diabetes melito e doença cardiovascular (10-14). Dois outros representantes dessa classe de antipsicóticos, risperidona e quetiapina, ofereceram riscos intermediários para ganho de peso e mostraram resultados discrepantes para avaliação do diabetes e da dislipidemia (15), enquanto a ziprasidona e o aripiprazol representam um risco menor. Uma metanálise realizada por Allison e cols. (16) mostrou que, dentro de dez semanas de tratamento, os pacientes em tratamento com antipsicóticos já apresentavam ganho de peso em comparação aos pacientes que recebiam placebo. Outros estudos mostram que as primeiras 12 semanas de uso de antipsicóticos são críticas para o ganho de peso (17). Esses dados demonstram que o ganho de peso é um fenômeno precoce no tratamento dos transtornos psicóticos.

Algumas explicações para o ganho de peso induzido por antipsicóticos seriam seus efeitos anticolinérgicos, anti-histaminérgicos, antagonismo dos receptores de serotonina, além da interferência da predisposição genética (18-22). O efeito anticolinérgico leva à boca seca, aumentando o consumo de líquidos calóricos que, por consequência, aumentam o peso (19). Os efeitos anti-histaminérgicos podem levar à sedação, diminuin- do a atividade e a movimentação, aumentando o peso consequentemente. $\mathrm{O}$ antagonismo $5 \mathrm{HT}_{2 \mathrm{~A}}$ leva ao aumento do consumo de alimentos calóricos, que também causam ganho de peso (18). Matsui-Sakata e cols. (20) também encontraram correlações entre os antipsicóticos por receptores $\mathrm{D}_{2}, 5 \mathrm{HT}_{1 \mathrm{~A}}, 5-\mathrm{HT}_{2 \mathrm{C}}$, receptores $\alpha 2$-adrenérgicos e o ganho de peso.

Segundo Nasrallah (22), a variação genética também influencia o ganho de peso induzido por antipsicóticos. Assim, uma variante no gene codificador para o receptor $5-\mathrm{HT}_{2 \mathrm{C}}$ foi associada à menor probabilidade de apresentar ganho de peso significativo após a terapia com olanzapina ou risperidona (22-25).

As intervenções que visam ao controle do ganho de peso podem ser farmacológicas ou não farmacológicas. Na revisão sistemática realizada por Faulkner (26), essas intervenções foram avaliadas, concluindo-se que a perda e/ou o controle de peso a curto prazo parecem possíveis com a prática regular de exercícios físicos e a orientação nutricional em conjunto com intervenções comportamentais. Intervenções farmacológicas têm sido usadas como apoio e devem ser a última opção terapêutica.

Sintomas como retraimento social, avolição, negativismo, ansiedade, comportamento impulsivo e desorganização do pensamento, muito presentes em pacientes com esquizofrenia, podem dificultar a adesão a um programa de exercícios ou o seguimento de uma dieta. Assim, para uma abordagem eficaz desses pacientes, é necessário um programa de prevenção de ganho de peso que atenda às suas especificidades, adequando atividade física e orientação nutricional às limitações impostas pela doença. Segundo Roeder (27), a atividade física desenvolvida em tais intervenções deve ter como objetivo a integração social e um melhor desempenho funcional dos pacientes nas atividades do cotidiano.

Vale ressaltar que, além da supressão dos sintomas psicóticos, o tratamento da esquizofrenia visa a melhorar a qualidade de vida para o paciente e sua inserção familiar e social, e a prevenir o estigma da doença $(28,29)$. É admissível esperar que intervenções que promovam um estilo de vida saudável ofereçam outros ganhos ao tratamento. Um programa integrado de orientação nutricional e atividade física tem um impacto positivo na qualidade de vida de pacientes portadores de transtornos mentais (30).

O objetivo deste artigo é apresentar uma revisão sobre os estudos controlados e não controlados de intervenções não farmacológicas para o manejo do ganho de peso em pacientes com esquizofrenia em tratamento com antipsicóticos. 


\section{ESTUDOS NÃO CONTROLADOS}

Aquila e Emanuel (31) realizaram um estudo com pacientes com transtornos mentais graves internados, no intuito de avaliar o efeito de uma orientação nutricional para controle do ganho de peso. Trinta e dois pacientes em tratamento com antipsicótico atípico há menos de um ano foram incluídos no estudo. Os pacientes recebiam uma dieta balanceada e participavam de um grupo composto por nutricionistas que os orientavam sobre alimentação saudável. Os pacientes foram pesados antes da intervenção e o peso foi reavaliado $12 \mathrm{e}$ 18 meses depois da intervenção. Trinta e um pacientes finalizaram o estudo. No início da intervenção, os pacientes tinham em média $88,6 \mathrm{~kg}( \pm 20,3 \mathrm{~kg})$. Após 12 meses, houve uma pequena redução no peso $(88,0 \mathrm{~kg} \pm$ $20,3 \mathrm{~kg}$ ), porém sem significância estatística. No início do estudo, $61 \%$ dos pacientes apresentaram ganho de peso, que foi reduzido para $31 \%$ um ano depois e para 43\% 18 meses depois, porém essa redução também não teve significância estatística. Apesar desses resultados, os autores concluem que o estudo é relevante, pois sinaliza a necessidade de intervenções nutricionais para prevenção e tratamento do ganho de peso associado ao uso de antipsicóticos.

Menza e cols. (32) realizaram um estudo não controlado com 31 pacientes em tratamento ambulatorial com esquizofrenia e transtorno esquizoafetivo em uso de antipsicóticos atípicos. Esses pacientes participaram de um programa de controle de ganho de peso durante um ano. A intervenção consistia em aconselhamento nutricional, exercícios e intervenções comportamentais planejadas para possibilitar mudanças no estilo de vida. Essas últimas incluíam o automonitoramento da alimentação e da atividade física, manejo do estresse, controle dos impulsos, resolução dos problemas e suporte social. Técnicas especiais para possíveis déficits cognitivos foram utilizadas, como repetição, tarefas para casa e uso de material visual. A comparação com um grupo-controle de 20 pacientes recebendo antipsicóticos atípicos, atendidos nos mesmos ambulatórios da pesquisa e no mesmo período de tempo mas sem a intervenção estudada, mostrou redução do peso de $3 \mathrm{~kg}$ no grupo que participou na intervenção, enquanto o grupo sem a intervenção ganhou em média $3,2 \mathrm{~kg}$ no período de um ano.

Kalarchian e cols. (33) realizaram um estudo com pacientes portadores de esquizofrenia ou transtorno esquizoafetivo com o IMC $\geq 30 \mathrm{~kg} / \mathrm{m}^{2}$. Trinta e cinco pacientes foram incluídos, $25(71,4 \%)$ deles em uso de clozapina. A intervenção foi conduzida por uma psicóloga e uma nutricionista e consistia em reuniões semanais com uma hora de duração durante 12 semanas, seguida por duas reuniões quinzenais e mais duas reuniões mensais. Durante o programa, trabalharam com um plano alimentar que dividia os alimentos em: verde (coma à vontade), amarelo (tome cuidado) e vermelho (evite), além de orientações com relação à prática de exercício. Foram avaliados peso, padrão alimentar (Eating Behavior Inventory), atividade física (Medical Outcome Study - MOS) e qualidade de vida (SF-36). Vinte e nove pacientes $(82,9 \%)$ completaram o tratamento semanal, participando, em média, de 10,6 $( \pm 1,7)$ reuniões. A média de perda de peso durante o tratamento foi de $2,28 \mathrm{~kg}( \pm 3,4)(\mathrm{t}=-3,6 \mathrm{l}, \mathrm{p}=$ 0,001 ), sendo que 20 pacientes perderam peso (entre 0,6 e 4,25 kg) e 9 ganharam peso (entre 0,09 e 4,25 $\mathrm{kg})$. Os autores também observaram que os $27(77,1 \%)$ pacientes que completaram as sessões extras (mensais) mantiveram a média de perda de peso de $3,23 \mathrm{~kg}( \pm$ $5,2)(\mathrm{t}=-3,23, \mathrm{p}=0,003)$, correspondendo a uma perda de $3,2 \%$ do peso inicial. Foi observado um resultado estatisticamente significativo comparando-se os escores totais da escala EBI $(\mathrm{t}=-4,66 ; \mathrm{p}=0,001)$, bem como a melhora na qualidade de vida em 3 das 8 subescalas: funcionamento físico $(\mathrm{t}=-3,55 ; \mathrm{p}=0,002)$, saúde em geral $(t=-2,07 ; p=0,049)$ e dor $(t=-2,56 ; p=0,017)$.

Em estudo realizado por Mauri e cols. (34), foi avaliada uma intervenção não farmacológica para prevenção e tratamento do ganho de peso associado ao uso de antipsicóticos, com duração de 12 semanas. Foram incluídos no estudo 53 pacientes que tiveram ganho de peso maior que $7 \%$ do peso inicial. Vinte e seis pacientes $(49 \%)$ finalizaram o estudo. No início, apresentavam um índice de massa corporal (IMC) de $31,9 \mathrm{~kg} / \mathrm{m}^{2}$ $\left( \pm 6,3 \mathrm{~kg} / \mathrm{m}^{2}\right)$, e depois de 12 semanas a média de IMC foi de $30,7 \mathrm{~kg} / \mathrm{m}^{2}\left( \pm 5,7 \mathrm{~kg} / \mathrm{m}^{2}, \mathrm{p}<0,001\right)$. Os autores destacaram que, apesar da perda de quase metade dos pacientes, o resultado poderia ser considerado positivo, pois a maioria daqueles que terminaram o estudo apresentou perda de peso estatisticamente significativa.

\section{ESTUDOS CONTROLADOS}

Alguns estudos adotaram essa modalidade para controle do peso em pacientes usando antipsicóticos. Setenta pacientes com esquizofrenia em uso de olanzapina participaram de uma intervenção em grupo com 16 sema- 
nas de duração (35). Os pacientes foram randomizados para intervenção ou tratamento usual. Os pacientes do grupo com intervenção perderam em média $0,2 \mathrm{~kg}$, e os controles ganharam 4,4 kg. A diferença encontrada foi atribuída ao maior conhecimento acerca de hábitos alimentares saudáveis e às maneiras de programar mudanças no estilo de vida.

Evans e cols. (36) realizaram um estudo controlado testando uma intervenção similar à de Littrell e cols. (35) em uma amostra de 51 pacientes com esquizofrenia em início de tratamento com olanzapina. Após três meses de estudo, os pacientes da intervenção ganharam $2 \mathrm{~kg}$ e os controles ganharam em média $6 \mathrm{~kg}(\mathrm{p}<0,002)$. A diferença foi mantida após seis meses de estudo $(2 \mathrm{~kg}$ versus $9,9 \mathrm{~kg}, \mathrm{p} \leq 0,013)$.

Brar e cols. (37) realizaram um estudo multicêntrico que comparou tratamento usual a uma intervenção comportamental em 72 pacientes com esquizofrenia ou transtorno esquizoafetivo. A intervenção consistia em 20 sessões durante 14 semanas, nas quais eram ensinadas diversas técnicas comportamentais para controle e perda de peso. $\mathrm{O}$ grupo submetido à intervenção perdeu em média $2,0 \pm 3,79 \mathrm{~kg}$, e o grupo em tratamento usual perdeu $1,1 \pm 3,11 \mathrm{~kg}$, mas essa diferença não foi estatisticamente significativa. Nove pacientes $(26,5 \%)$ do grupo de intervenção e $4(10,8 \%)$ do grupo controle apresentaram perda de peso maior ou igual a $5 \%$ do peso inicial $(\mathrm{p}=0,82)$. Além disso, os autores perceberam uma satisfação maior com o tratamento nos pacientes submetidos à intervenção, avaliada pelo Client Satisfaction Questionnaire (CSQ-8), $\mathrm{p}=0,015$.

Outro estudo controlado foi realizado com $61 \mathrm{pa}-$ cientes de primeiro episódio psicótico em uso de risperidona, olanzapina e haloperidol (38). Os autores compararam uma intervenção precoce com cuidados físicos não estruturados na prevenção do ganho de peso desses pacientes. A intervenção foi oferecida durante três meses e objetivou ensinar estratégias para aumentar o controle sobre fatores associados ao ganho de peso, como ingestão calórica e atividade física. Ao final do estudo, ambos os grupos ganharam peso, mas o grupo da intervenção ganhou significativamente menos peso do que o grupo-controle $(4,1 \pm 4 \mathrm{~kg}$ versus $6,9 \pm 4,5 \mathrm{~kg}$, $\mathrm{p}<0,01)$.

Kwon e cols. (39) realizaram ensaio clínico randomizado, multicêntrico controlado, no qual 48 pacientes com esquizofrenia ou transtorno esquizoafetivo em uso de olanzapina foram randomizados para uma intervenção de 12 semanas com conteúdo principal sobre dieta e exercício físico. Houve redução de peso e IMC estatisticamente significativo nos pacientes submetidos à intervenção quando comparados aos controles.

Weber e Wyne (40) conduziram um estudo piloto com 17 pacientes com diagnóstico de esquizofrenia ou transtorno esquizoafetivo que apresentavam IMC $\geq 25 \mathrm{~kg} / \mathrm{m}^{2}$. Os pacientes foram randomizados: nove foram alocados para tratamento convencional e oito para um grupo com enfoque cognitivo comportamental - Diabetes Prevention Project (DPP) - com reuniões semanais de uma hora durante 16 semanas. Foram avaliados peso, IMC, relação cinturaquadril e glicemia. As sessões foram realizadas por uma enfermeira treinada por duas semanas. Quinze pacientes $(88,23 \%)$ finalizaram o estudo, sete do grupo-controle e oito da intervenção. Não houve diferenças estatisticamente significativas com relação ao peso, ao IMC, à relação cintura quadril e à glicemia nos pacientes antes e depois da intervenção.

Scocco e cols. (41) realizaram um estudo para avaliar a eficácia de uma intervenção psicoeducacional para orientação nutricional de pacientes portadores de esquizofrenia e em uso de olanzapina. Os pacientes que estavam no início do tratamento com olanzapina foram randomizados para dois grupos: $\mathrm{O}$ grupo 1, com 9 pacientes inseridos em uma intervenção psicoeducacional para controle do ganho de peso, de 24 semanas, e o grupo 2, com 8 pacientes que não foram incluídos na intervenção nas primeiras oito semanas, apenas nas 16 semanas finais. Foram avaliados peso e suas variações nos últimos três meses, uso prévio de antipsicótico convencional, adesão a tratamentos anteriores, tabagismo e foi utilizada a escala Global Clinical Judgement (GCJ) para avaliar a gravidade da doença. Os dados foram coletados antes da intervenção, 8 e 24 semanas depois. $\mathrm{Na}$ avaliação da oitava semana, não foram observadas alterações de peso nos pacientes do grupo $\mathrm{l}(+0,99$ $\pm 3,34 \mathrm{~kg} ; \mathrm{t}=0,89, \mathrm{df}=8, \mathrm{p}=0,4)$, enquanto os pacientes do grupo 2 apresentaram um aumento significativo no peso $(+2,96 \pm 3,08 \mathrm{~kg} ; \mathrm{t}=2,72 ; \mathrm{df}=7, \mathrm{p}<$ $0,03)$. No final do estudo (24 semanas), todos os pacientes apresentaram aumento de peso $(+2,36 \pm 4,01$ $\mathrm{kg} ; \mathrm{t}=2,43, \mathrm{df}=16, \mathrm{p}<0,03)$, no entanto, na avaliação intermediária (entre a $8^{\mathrm{a}}$ e $24^{\mathrm{a}}$ semana), os pacientes do grupo 2 apresentaram uma diminuição do peso $(-1,77$ $\pm 1,71 \mathrm{~kg} ; \mathrm{t}=-2,93, \mathrm{df}=7, \mathrm{p}<0,03)$, comparados aos 
pacientes do grupo $\mathrm{l}(3,16 \pm 3,59 \mathrm{~kg} ; \mathrm{t}=2,63, \mathrm{df}=8$, $\mathrm{p}<0,03)$. O aumento do peso foi maior no grupo $\mathrm{l}$ $(+3,4 \pm 4,39 \mathrm{~kg} ; \mathrm{t}=2,32, \mathrm{df}=8, \mathrm{p}<0,05)$ do que no grupo $2(+1,19 \pm 3,42 \mathrm{~kg} ; \mathrm{t}=0,98, \mathrm{df}=7, \mathrm{p}=0,36)$ com relação às medidas iniciais e finais.

Khazaal e cols. (42) compararam uma intervenção cognitivo-comportamental de 12 semanas de duração conhecida por Apple pie group, com uma intervenção nutricional breve (INB), de duas horas de duração. A avaliação dos pacientes acontecia no início, nas semanas 12 e 24 . Sessenta e um pacientes foram randomizados e, além do peso e IMC, foram avaliadas as cognições ligadas ao peso e à presença de compulsão alimentar. Os pacientes da intervenção comportamental apresentaram melhora da sintomatologia da compulsão alimentar e das cognições ligadas ao peso e uma maior perda de peso em 24 semanas quando em comparação aos pacientes submetidos à INB.

Álvarez-Jiménez e cols. (30) realizaram revisão sistemática e metanálise sobre intervenções não farmacológicas para manejo do ganho de peso ligado ao uso de antipsicóticos. Vinte e oito estudos controlados foram avaliados, mas apenas dez foram incluídos no estudo. Seis estudos investigaram intervenções cognitivo-comportamentais, três avaliaram aconselhamento nutricional e um combinou intervenção nutricional e exercício físico. Cinco estudos controlados testaram intervenções em grupo, e cinco eram individuais. Quatro estudos objetivaram a prevenção do ganho de peso induzido por antipsicóticos e seis intervieram em pacientes que já haviam ganho peso. As intervenções duraram entre oito semanas e seis meses. Ao todo, os dez estudos envolveram 482 pacientes; houve redução estatisticamente significativa do peso dos pacientes submetidos às intervenções quando comparados ao tratamento usual $(-2,56 \mathrm{~kg}$; IC: $-3,2$ a $-1,92 \mathrm{~kg} ; \mathrm{p}<0,001)$. O IMC também apresentou redução estatisticamente significativa. Não houve diferença entre as abordagens terapêuticas (individual versus grupo, aconselhamento nutricional versus cognitivo-comportamental).

Em resumo, o conjunto dos estudos sugere que as medidas não farmacológicas para controle do peso podem ser efetivas em pacientes com esquizofrenia (Tabela 1).

\section{COMENTÁRIOS E PERSPECTIVAS}

Ganho de peso é uma preocupação importante em pacientes psicóticos em razão do risco de diabetes melito e doenças cardiovasculares. Medidas não farmacológicas podem ser uma ferramenta eficaz na prevenção e no controle do ganho de peso, podendo levar à redução da morbimortalidade relacionada aos distúrbios metabólicos secundários ao ganho de peso nessa população (43). Entre as intervenções para ganho de peso, dá-se preferência às não farmacológicas, mas, ainda assim os resultados esperados podem não ser satisfatórios. Nos casos em que o paciente tem dificuldade em aderir às novas propostas de hábitos saudáveis, o tratamento medicamentoso para perda de peso pode ser eficaz, no entanto, sempre deve estar associado às estratégias para mudança de estilo de vida. O orlistat não tem absorção sistêmica, não interagindo com os antipsicóticos no sistema nervoso central, porém requer adesão do paciente a um regime de baixa ingestão de gorduras (44). Estudos controlados realizados com topiramato têm mostrado eficácia desse medicamento na redução de peso em pacientes com esquizofrenia $(45,46)$, além de não alterar significativamente as concentrações de clozapina, olanzapina, risperidona e quetiapina quando administrado em doses anticonvulsivantes (47).

No estudo realizado por James e cols. (48), os resultados com a utilização de sibutramina em pacientes obesos foram satisfatórios na perda de peso, além de proporcionarem a redução de triglicérides e o aumento de colesterol HDL $(48,49)$. Em estudos controlados com pacientes em uso de antipsicóticos, o uso de sibutramina comparado ao placebo mostrou resultados conflitantes que podem estar relacionados ao tamanho da amostra. Entretanto, demonstrouse a segurança dessa medicação nos pacientes com esquizofrenia, não induzindo sintomas psicóticos $(48,50)$.

A droga que tem mostrado resultados mais consistentes na redução de peso em pacientes esquizofrênicos em tratamento com antipsicóticos de segunda geração é a metformina, antidiabético oral muito eficaz no controle de diabetes tipo 2 com obesidade, mas que também tem sido empregada em outras condições que cursam com resistência à insulina como no pré-diabetes e na síndrome dos ovários policísticos $(51,52)$. Vale ressaltar que a hiperglicemia é um efeito colateral associado ao uso de antipsicóticos nos indivíduos predispostos, e essa medicação tem efeito protetor para a evolução do diabetes tipo $2 \mathrm{em}$ indivíduos de risco (52). 


\begin{tabular}{|c|c|c|c|c|c|c|}
\hline Estudo & $\mathbf{n}$ & Técnica & Controle & Abandono (\%) & Duração & Resultados \\
\hline Aquila e Emanuel (31) & 31 & $\begin{array}{l}\text { Nutrição + exercício + } \\
\text { dieta de } 2.000 \text { calorias } \\
\text { (pacientes internados) }\end{array}$ & Não & 3 & 18 meses & $\downarrow 0,6 \mathrm{~kg} 18$ meses depois \\
\hline Littrell e cols. (35) & 70 & Nutrição + exercício & $\operatorname{Sim}^{*}$ & Não & 6 meses & $\begin{array}{l}\text { Intervenção: -0,02 kg } \\
\text { Controle: }+4 \text { kg }\end{array}$ \\
\hline Menza e cols. (32) & 31 & $\begin{array}{l}\text { Nutrição + exercício + } \\
\text { comportamental }\end{array}$ & $\operatorname{Sim}^{\star}$ & 32,3 & $\begin{array}{c}12 \text { semanas }+6 \text { meses } \\
\text { manutenção }\end{array}$ & $\begin{array}{l}\text { Intervenção: }-3 \text { kg } \\
\text { Controle: }+3,2 \text { kg }\end{array}$ \\
\hline Evans e cols. (36) & 51 & $\begin{array}{l}\text { Nutrição + exercício + } \\
\text { comportamental }\end{array}$ & $\operatorname{Sim}^{*}$ & 33,3 & 12 semanas & $\begin{array}{l}\text { Intervenção: }+2 \text { kg } \\
\text { Controle: }+6 \text { kg }\end{array}$ \\
\hline Brar e cols. (37) & 72 & Técnica comportamental & $\operatorname{Sim}^{*}$ & 30 & 14 semanas & $\begin{array}{c}\text { Intervenção: -2 kg } \\
\downarrow \text { PAS } \\
\text { Controle: }-1,1 \mathrm{~kg}\end{array}$ \\
\hline Kalarchian e cols. (33) & 35 & Dieta cores + comportamental & Não & 17 & $\begin{array}{c}12 \text { semanas + } \\
\text { manutenção } 3 \text { meses }\end{array}$ & $-2,28 \mathrm{~kg}$ \\
\hline Scocco e cols. (41) & 17 & Psicoeducação & Sim & 15 & 24 semanas & $\begin{array}{l}\text { Intervenção } 24 \text { sem. +3,4 kg } \\
\text { Intervenção } 16 \text { sem. -1,19 kg }\end{array}$ \\
\hline Weber e Wyne (40) & 17 & Técnica comportamental & $\operatorname{Sim}^{*}$ & 11,8 & 16 semanas & $\begin{array}{c}\text { Não encontraram alterações } \\
\text { significantes }\end{array}$ \\
\hline $\begin{array}{l}\text { Álvarez-Jimenez e cols. } \\
\text { (38) }\end{array}$ & 61 & $\begin{array}{l}\text { Nutrição + exercício + } \\
\text { comportamental }\end{array}$ & $\operatorname{Sim}^{*}$ & Não & 12 semanas & $\begin{array}{l}\text { Intervenção: }+4,1 \text { kg } \\
\text { Controle: }+6,9 \text { kg }\end{array}$ \\
\hline Kwon e cols. (39) & 48 & $\begin{array}{l}\text { Individual } \\
\text { diário alimentar + } \\
\text { atividade física }\end{array}$ & $\operatorname{Sim}^{*}$ & 25 & 12 semanas & $\begin{array}{l}\text { Intervenção: }-3,9 \text { kg } \\
\text { Controle: }-1,48 \mathrm{~kg}\end{array}$ \\
\hline Mauri e cols. (34) & 53 & Dieta + atividade física (4 sessões) & Não & 51 & 12 semanas & $-3,15 \mathrm{~kg}$ \\
\hline Khazaal e cols. (42) & 61 & TCC x educação nutricional breve & $\operatorname{Sim}^{*}$ & 13,1 & 12 semanas & $\begin{array}{l}\text { Intervenção: -1,68 kg } \\
\text { Controle: }-0,52 \mathrm{~kg}\end{array}$ \\
\hline
\end{tabular}

* Estudos randomizados; TCC: terapia cognitivo-comportamental; PAS: pressão arterial sistólica.

As demais medicações têm ação no sistema nervoso central e podem interferir no tratamento da esquizofrenia. A utilização de algumas medicações pode contribuir para o tratamento do ganho de peso, no entanto, a combinação entre técnicas de manejo de peso de forma flexível e que leve em conta a realidade dos serviços de saúde e as necessidades individuais parece produzir melhores resultados.

Apesar de existirem poucos estudos, as intervenções para prevenção do ganho de peso em usuários de antipsicóticos parecem melhores do que quando o ganho de peso já se instalou. Uma limitação da maioria dos estudos levantados é o curto período de acompanhamento, muitas vezes insuficiente para que mudanças sejam observadas. Alguns estudos comentam que o tamanho amostral é pequeno $(36,39,41)$. Estudos como o de Littrell e cols. (35) não controlaram as medicações concomitantes, o que sabemos ser uma limitação devido ao fato de as medicações apresentarem perfis diferentes em relação ao ganho de peso. $\mathrm{O}$ fato de o grupo-controle de alguns estudos não ser submetido a nenhum tipo de tratamento (42) também é uma limitação na avaliação da eficácia de tais intervenções.

Os autores participam de um projeto multicêntrico nacional que, atualmente, envolve 125 serviços de saúde mental com o objetivo de avaliar os efeitos de uma intervenção não farmacológica para controle do ganho de peso em pacientes com transtornos mentais graves, o Programa Bem-Estar (53).

O Programa Bem-Estar foi realizado em 57 serviços de saúde mental em todo o Brasil. Peso ( $\mathrm{kg}$ ), cálculo do índice de massa corporal (IMC): (peso/quadrado da altura, $\left.\mathrm{kg} / \mathrm{m}^{2}\right)$, cintura $(\mathrm{cm})$, pressão arterial $(\mathrm{mmHg})$ e prática de atividade física foram registrados no início e no final do programa. Seiscentos e cinquenta e seis pacientes já participaram do Programa. Após a intervenção, os pacientes apresentaram uma perda estatisticamente significativa de $0,53 \pm 3,4 \mathrm{~kg}$ (média \pm 
DP, p $<0,001)$, e redução de IMC de $0,2 \pm 1,4$ $\mathrm{kg} / \mathrm{m}^{2}$ (média $\pm \mathrm{DP}, \mathrm{p}<0,00 \mathrm{l}$ ). Houve aumento da atividade física após a intervenção: no início, 290 $(55,2 \%)$ pacientes realizavam algum tipo de atividade física e, após a intervenção, esse número foi para $382(72,8 \%)$ (McNemar test, $\mathrm{p}<0,001)$.

Para avaliar o impacto da intervenção, atualmente está se conduzindo um ensaio clínico randomizado, multicêntrico, comparando a intervenção e a lista de espera. Dessa forma, pretende-se contribuir nessa área testando intervenções que sejam úteis para a nossa realidade.

Declaração: Cecília Attux: recebeu verba de pesquisa da Fapesp e Eli Lilly; Larissa Campagna Martini: recebeu verba de pesquisa da Fapesp, Eli Lilly e Capes; Rodrigo A. Bressan: speaker e membro do board da Janssen, Eli Lilly, AstraZeneca, Organon, Novartis. Verbas de pesquisa da Fapesp, CNPq e Capes. André F. Reis: declara não haver conflitos de interesse científico neste estudo.

\section{REFERÊNCIAS}

1. American Diabetes Association, American Psychiatric Association. Consensus development conference on antipsychotic drugs and obesity and diabetes. J Clin Psychiatry. 2004;65(2):267-72.

2. Mukherjee S, Decina P, Bocola V, Saraceni F, Scapicchio PL. Diabetes mellitus in schizophrenic patients. Compr Psychiatry. 1996;37(1):68-73.

3. Nasrallah H. High prevalence of diabetes mellitus in schizophrenia, schizoaffective disorder, and bipolar disorder. Int J Neuropsychopharmacol. 2000;3(Suppl 1):S116.

4. Strauss J, Rakfeldt J, Harding CM, Lieberman P, et al. Psychological and social aspects of negative symptoms. Br J Psychiatry. 1989;155(Suppl 7):128-32.

5. Sartorius N. One of the last obstacles to better mental health care: the stigma of mental illness. In: Guimón J, FischerW, Sartorius N, editors. The image of madness: the public facing mental illness and psychiatric treatment. Genebra: Karger. 1999. p. 96-104.

6. Elkis H, Gama C, Suplicy H, Tambascia M, Bressan R, Lyra R, et al. Consenso Brasileiro sobre antipsicóticos de segunda geração e distúrbios metabólicos. Rev Bras Psiquiatr. 2008;30 (1):77-85.

7. Almeida B, Ferreira SRG. Epidemiologia. In: Claudino AM, Zanella MT. Transtornos alimentares e obesidade. Barueri, SP: Manole; 2005. p. 185-93.

8. Weinsier RL, Hunter GR, Heini AF, Goran MI, Sell SM. The etiology of obesity: relative contribution of metabolic factors, diet, and physical activity. Am J Med. 1998;105(2):145-50.

9. Bressan RA, Erlandsson $K$, Jones HM, Mulligan RM, Flanaghan R, Ell PJ, et al. Selective D2/D3 dopamine receptor occupancy is sufficient for atypical antipsychotic effect. An in vivo quantitative [123I]-epidepride single photon emission tomography (SPET) study of amisulpride treated patients. Am J Psychiatry. 2003;160(8):1413-20.

10. Nasrallah H. A review of the effect of atypical antipsychotics on weight. Psychoneuroendocrinology. 2003;28(Suppl 1):83-96.

11. Meyer JM. A retrospective comparison of weight, lipid, and glucose changes between risperidone- and olanzapine-treated inpatients: metabolic outcomes after 1 year. J Clin Psychiatry. 2002;63(5):425-33.
12. Sernyak MJ, Leslie DL, Alarcon RD, Losonczy MF, Rosenheck R. Association of diabetes mellitus with use of atypical neuroleptics in the treatment of schizophrenia. Am J Psychiatry. 2002;159(4):561-6.

13. Reis AF. Antipsychotic drugs and metabolic syndrome: can we prevent it? Rev Bras Psiquiatr. 2007;29(1):9-10.

14. Attux C, Quintana MI, Chaves AC. Weight gain, dyslipidemia and altered parameters for metabolic syndrome on first episode psychotic patients after six-month follow-up. Rev Bras Psiquiatr. 2007;29(4):346-9.

15. Attux $C$, Chaves AC. New onset diabetes associated with quetiapine treatment: case report. Rev Bras Psiquiatr. 2005;27(3):254.

16. Allison DB, Mentore JL, Heo M, Chandler LP, Cappelleri JC, Infante $\mathrm{MC}$, et al. Antipsychotic-induced weight gain: a comprehensive research synthesis. The American journal of psychiatry. 1999;156(11):1686-96.

17. Kinon BJ, Basson BR, Gilmore JA, Tollefson GD. Long-term olanzapine treatment: weight change and weight-related health factors in schizophrenia. J Clin Psychiatry. 2001;62(2):92-100.

18. Wirshing DA, Wirshing WC, Kysar L, Berisford MA, Goldstein D, Pashdag J, et al. Novel antipsychotics: comparison of weight gain liabilities. J Clin Psychiatry. 1999;60(6):358-63.

19. Wetterling T. Bodyweight gain with atypical antipsychotics. A comparative review. Drug Saf. 2001;24(1):59-73.

20. Matsui-Sakata A, Ohtani H, SawadaY. Receptor occupancy-based analysis of the contributions of various receptors to antipsychotics-induced weight gain and diabetes mellitus. Drug Metab Pharmacokinet. 2005;20(5):368-78.

21. Kim SF, Huang AS, Snowman AM, Teuscher C, Snyder SH. From the cover: antipsychotic drug-induced weight gain mediated by histamine $\mathrm{H} 1$ receptor-linked activation of hypothalamic AMPkinase. Proc Natl Acad Sci U S A. 2007;104(9):3456-9.

22. Nasrallah HA. Atypical antipsychotic-induced metabolic side effects: insights from receptor-binding profiles. Mol Psychiatry. 2008;13(1):27-35.

23. Reynolds GP, Zhang ZJ, Zhang XB. Association of antipsychotic drug-induced weight gain with a $5-\mathrm{HT} 2 \mathrm{C}$ receptor gene polymorphism. Lancet. 2002;359(9323):2086-7.

24. Templeman LA, Reynolds GP, Arranz B, San L. Polymorphis$\mathrm{ms}$ of the 5-HT2C receptor and leptin genes are associated with antipsychotic drug-induced weight gain in caucasian subjects with a first-episode psychosis. Pharmacogenet Genomics. 2005;15(4):195-200.

25. Lane HY, Liu YC, Huang CL, Chang YC, Wu PL, Lu CT, et al. Risperidone-related weight gain: genetic and nongenetic predictors. $\mathrm{J}$ Clin Psychopharmacology. 2006;26(2):128-34.

26. Faulkner G, Soundy AA, Lloyd K. Schizophrenia and weight management: a systematic review of interventions to control weight. Acta Psychiatr Scand. 2003;108(5):324-32.

27. Roeder MA. Atividade física, saúde mental e qualidade de vida. Rio de Janeiro: Shape; 2003.

28. Bressan RA, Costa DC, Jones HM, Ell PJ, Pilowsky LS. Typical antipsychotic drugs: D2 receptor blockade and depressive symptoms in schizophrenia. Schizophr Res. 2002;56(1-2):31-6.

29. Bressan RA, Chaves AC, Pilowsky LS, Shirakawa I, Mari JJ. Depressive Episodes in Stable Schizophrenia: critical evaluation of the DSM-IV and CID-10 diagnostic criteria. Psychiatry Res. 2003;117(1):47-56.

30. Álvarez-Jiménez $M$, Hetrick SE, González-Blanch C, Gleeson JF, McGorry PD. Non-pharmacological management of antipsychotic-induced weight gain: systematic review and meta-analysis of randomized controlled trials. Br J Psychiatry. 2008;193(2):101-7.

31. Aquila R, Emanuel M. Interventions for weight gain adults treated with novel Aatipsycotics. J Clin Psychiatry. 2000;2(1):20-3. 
32. Menza M, Vreeland B, Minsky S, Gara M, Radler DR, Sakowitz M. Managing Atypical antipsychotic-associated weight gain: 12month data on a multimodal weight control program. J Clin Psychiatry. 2004;65(4):471-7.

33. Kalarchian MA, Marcus MD, Levine MD, Haas GL, Greeno CG, Weissfeld LA, et al. Behavioral treatment of obesity in patients taking antipsychotic medications. J Clin Psychiatry. 2005;66(8):1058-63.

34. Mauri M, Castrogiovanni S, Simoncini M, lovieno N, Miniati M, Rossi $A$, et al. Effects of an educational intervention on weight gain in patients treated with antipsychotics. J. Clin Psychopharmacology. 2006;26(5):462-6.

35. Littrell KH, Hilligoss NM, Kirshner CD, Petty RG, Johnson CG. The effects of an educational intervention on antipsychotic-induced weight gain. J Nurs Scholarsh. 2003;35(3):237-41.

36. Evans $S$, Newton $R$, Higgins $S$. Nutritional intervention to prevent weight gain in patients commenced on olanzapine: a randomized controlled trial. Aust N Z J Psychiatry. 2005;39(6):479-86.

37. Brar JS, Ganguli R, Pandina G, Turkoz I, Berry S, Mahmoud R. Effects of behavioral therapy on weight loss in overweight and obese patients with schizophrenia or schizoaffective disorder. J Clin Psychiatry. 2005;66 (2):205-12.

38. Álvarez-Jiménez $M$, González-Blanch $C$, Vázquez-Barquero JL, Pérez-Iglesias R, Martínez-Garcia O, Pérez-Pardal T, et al. Attenuation of antipsychotic-induced weight gain with early behavioral intervention in drug-naive first-episode psychosis patients: a randomized controlled trial. J Clin Psychiatry. 2006;67(8):1253-60.

39. Kwon JS, Choi JS, Bahk WM, Kim CY, Kim CH, Shin YC, et al. Weight management program for treatment-emergent weight gain in olanzapine-treated patients with schizophrenia or schizoaffective disorder: a 12-week randomized controlled clinical trial. J Clin Psychiatry. 2006;67(4):547-53.

40. Weber M, Wyne K. A cognitive/behavioral group intervention for weight loss in patients treated with atypical antipsychotics. Schizophr Res. 2006;83(1):95-101.

41. Scocco $\mathrm{P}$, Longo $\mathrm{R}$, Caon F. Weight change in treatment with olanzapine and a psychoeducational approach. Eating Behaviors. 2006;7(2):115-24.

42. Khazaal Y, Fresard E, Rabia S, Chatton A, Rothen S, Pomini V, et al. Cognitive behavioral therapy for weight gain associated with antipsychotic drugs. Schizophr Res. 2007;91(1-3):169-77.
43. Reis JS, Alvarenga T, Rosário PW, Menezes PA, Rocha R dos S. Diabetes mellitus associado com drogas antipsicóticas atípicas: relato de caso e revisão da literatura. Arq Bras Endocrinol Metab. 2007;51(3):488-93.

44. Mancini MP, Halpern A. Pharmacological treatment for obesity. Arq Bras Endocrinol Metabol. 2006;50(2):377-89.

45. KoYH, Joe SH, Jung IK, Kim SH. Topiramate as an adjuvant treatment with atypical antipsychotics in schizophrenic patients experiencing Weight gain. Clin Neuropharmacol. 2005;28(4):169-75.

46. Egger $C$, Muehlbacher M, Schatz M, Nickel M. Influence of topiramate on olanzapine-related weight gain in women: an 18-month follow-up observation. J Clin Psychopharmacol. 2007;27(5):475-8.

47. Lévy E, Agbokou C, Ferreri F, Chouinard G, Margolese HC. Topiramate-induced weight gain in schizophrenia: a retrospective case series study. Can J Clin Pharmacol. 2007;14(2):e234-9.

48. James WP, Astrup A, Finer N, Hilsted J, Kopelman P, Rossner S, et al. Effect of sibutramine on weight maintenance after weight loss: a randomized trial. STORM Study Group (Sibutramine Trial of Obesity Reduction and Maintenance). Lancet. 2000;356 (9248):2119-25.

49. Rucker D, Padwal R, Li SK, Curioni C, Lau DCW, et al. Long term pharmacotherapy for obesity and overweight: updated metaanalysis. BMJ. 2007;335 (7631):1194-9.

50. Henderson DC, Fan X, Copeland PM, Borba CP, DaleyTB, Nguyen $\mathrm{DD}$, et al. A double-blind, placebo-controlled trial of sibutramine for clozapine-associated weight gain. Acta Psychiatr Scand. 2007;115(2):101-5.

51. Wu RR, Zhao JP, Guo XF, HeYQ, Fang MS, et al. Metformin addition attenuates olanzapine-induced weight gain in drug-naïve first-episode schizophrenia patients: a double-blind, placebocontrolled study. Am J Psychiatry. 2008;165(3):352-8

52. Bailey CJ, Turner RC. Metformin. N Engl $J$ Med. 1996;334(9):574-9.

53. Attux C, Martini LC, Araujo CM, Roma AM, Mullan EM, et al. Nonpharmacological management of weight gain: a national, multicentric study for schizophrenia and severe mental disorders In: XIV Biennial Winter Workshop on Schizophrenia and Bipolar Disorders, 2008, Montreux. Schizoph Res. Amsterdam: Elsevier; 2008;98:52-52. 